\title{
Systematic Construction of Abstractions for Model-Checking
}

\author{
Arie Gurfinkel, Ou Wei, and Marsha Chechik \\ Department of Computer Science, University of Toronto \\ Email: $\{$ arie, owei, chechik\}@cs.toronto.edu
}

This paper describes a framework, based on Abstract Interpretation, for creating abstractions for model-checking. Specifically, we study how to abstract models of $\mu$ calculus and systematically derive abstractions that are constructive, sound, and precise, and apply them to abstracting Kripke structures. The overall approach is based on the use of bilattices to represent partial and inconsistent information.

\section{Introduction}

Abstraction plays a fundamental role in combating state-space explosion in modelchecking. The goal of abstraction is to construct an abstract model of a system which is small enough to be effectively analyzed, and yet rich enough to yield conclusive results. Success of current abstraction projects, such as SLAM [2] and Bandera [6], indicates that abstraction is an effective technique for enabling model-checking of realistic software systems.

In model-checking, a notion of abstracting a transition system is typically developed as follows: (1) An abstract statespace is defined such that each abstract state corresponds to a set of concrete states. This correspondence can be arbitrary, as in predicate abstraction [17], or influenced by the concrete statespace, as in symmetry reduction [12]. (2) An abstract transition system is constructed by defining a transition relation over this abstract statespace. (3) Finally, the resulting system is argued to be sound, i.e., it is shown to preserve a fragment of the desired temporal logic.

The problem with the above approach is that it is not algorithmic: the techniques used to construct the abstract systems require a certain amount of intuition of users, and extra effort is needed to show that the resulting abstraction is correct. This makes it difficult to understand a specific abstraction method and improve on it. For example, given an abstraction that preserves universal CTL, how should it be changed to preserve the entire CTL? It is also difficult to understand the relationship between different abstract methods. For example, as shown in [25], predicate abstraction and symmetry reduction differ only in their choice of abstract states. However, this insight was not apparent just from the description of these methods.

Given the role abstraction plays in the model-checking process, we believe it is essential to create a general methodology for systematically constructing and analyzing abstractions. In the context of static analysis of programs, such a framework, called Abstract Interpretation (AI), has already been proposed by [7]. It provides a collection of notations and tools to formalize the approximation of program semantics, as well as to design and analyze program abstractions. The goal of this paper is to specialize the AI framework to model-checking. 
There are a number of ways to do this specialization, given the breadth of modelchecking approaches. Our goal here is to create abstractions that preserve properties expressed in the modal $\mu$-calculus [20] $\left(L_{\mu}\right)$. Following the recipes of AI, we systematically derive conditions under which an abstract $L_{\mu}$ model is the best abstraction of a concrete one. We guarantee that these abstract models are (a) sound, i.e., if an $L_{\mu}$ formula is satisfied in the abstract model it is satisfied in the concrete, (b) most precise, i.e., satisfy the most properties, and (c) have the desired structural characteristics, e.g., a requirement that an abstraction of a transition system is a transition system as well. These conditions are constructive and, as we show in this paper, can be derived almost mechanically. The algorithm for building a desired abstraction follows from these conditions directly.

The logic $L_{\mu}$ includes negation, so that an $L_{\mu}$ formula $\neg \varphi$ is satisfied iff $\varphi$ is refuted. If we assume that every formula is either satisfied or refuted in an abstraction as well, it may seem that preserving soundness for all $L_{\mu}$ formulas means that such an abstraction must satisfy and refute exactly the same properties as the corresponding concrete model (resulting in a bisimilar model). If the goal is to save space for model-checking, this abstraction would be very limited. Thus, most existing abstractions are restricted to fragments of $L_{\mu}$, i.e., only to the universal or only to the existential properties (see, e.g., [22]).

The insight we use in this paper is that an abstraction is inherently incomplete: some formulas may be neither satisfied nor refuted by it. We propose to treat satisfaction and refutation independently. If we classify all $L_{\mu}$ formulas using a pair $\langle$ Sat, Ref $\rangle$, where Sat contains all the formulas satisfied in an abstract model, while Ref contains all the refuted ones, then Sat and Ref are not necessarily complements of each other. In fact, Sat and Ref may not even be disjoint, allowing some formulas to be both satisfied and refuted.

Associating knowledge about truth and falsity of every piece of evidence can be naturally encoded using 4-valued Belnap logic [3] which enjoys nice mathematical properties associated with bilattices $[15,14]$. That is, bilattices enable a uniform approach for handling partial and inconsistent information, allowing reasoning about truth and knowledge in a single theoretical framework. In this paper, by combining the theories of AI with that of bilattices, we obtain a simple and elegant framework for deriving abstractions for $L_{\mu}$. Due to the generality of bilattices, our results apply not only to the traditional two-valued interpretation of $L_{\mu}$, but also to its multi-valued [4] and quantitative [10] interpretations.

The contribution of this paper is a general technique, based on AI, for deriving abstractions for model-checking. It allows understanding and comparing different techniques, and provides a methodology for proving soundness and precision of the desired abstraction. We then study this technique on two additional levels. First, we apply it to $L_{\mu}$ models, and then specialize it to abstracting transition systems represented as Kripke structures.

The rest of this paper is organized as follows: after providing the necessary background in Section 2, we show how to lift abstraction between elements to abstraction between sets of elements in Section 3. This gives us a general framework for approximating interpretations of $L_{\mu}$. In Section 4, we derive abstractions for model-theoretic 
interpretations of $L_{\mu}$, and then apply our technique to abstracting transition systems in Section 5. In Section 6, we specialize the results of Section 5 to boolean transition systems, and compare them to those obtained by Dams et al. [8]. We relate our technique with other abstraction approaches in Section 7 and summarize our contributions in Section 8.

\section{Background}

In this section, we introduce the basic concepts of lattice theory, modal $\mu$-calculus, and abstract interpretation.

\subsection{Lattices and Monotone Functions}

A lattice is a partially ordered set $\mathcal{L}=(L, \leq)$ in which every subset $B$ of $L$ has a least upper bound, called join and denoted $\sqcup B$, and a greatest lower bound, called meet and denoted $\sqcap B$ [9]. A lattice is distributive if meet and join distribute over each other, i.e., $(a \sqcap b) \sqcup c=(a \sqcup c) \sqcap(b \sqcup c)$, and $(a \sqcup b) \sqcap c=(a \sqcap c) \sqcup(b \sqcap c)$.

A De Morgan algebra is a structure $\mathcal{D}=(L, \leq,-)$, where $(L, \leq)$ is a distributive lattice and $-: L \rightarrow L$ is a negation that satisfies involution $(--a=a)$ and De Morgan laws: $-(a \sqcap b)=-a \sqcup-b$, and $-(a \sqcup b)=-a \sqcap-b$.

We denote the space of functions from $A$ to $B$ by $A \rightarrow B$, or $B^{A}$. For example, both $A \rightarrow[B \rightarrow C]$ and $\left(C^{B}\right)^{A}$ denote the space of functions from $A$ to functions from $B$ to $C$.

Let $A$ be a set and $\mathcal{L}=(L, \leq)$ be a lattice. The ordering and operations of $\mathcal{L}$ extend pointwise to $L^{A}$, i.e., $f \leq g \Leftrightarrow \forall a \in A \cdot f(a) \leq g(a)$. This turns $L^{A}$ into a lattice with the same properties as $\mathcal{L}$. In particular, if $\mathcal{L}$ is distributive or De Morgan, so is $L^{A}$.

A function $f$ between two partially ordered sets $(A, \leq)$ and $(B, \sqsubseteq)$ is monotone (or, order-preserving) iff $a \leq b \Rightarrow f(a) \sqsubseteq f(b)$, and anti-monotone iff $a \leq b \Rightarrow$ $f(b) \sqsubseteq f(a)$. We use upward $(\uparrow)$ and downward $(\downarrow)$ arrows to indicate monotone and anti-monotone functions, respectively. For example, $[A \rightarrow B]_{\uparrow}$ denotes the space of all monotone functions from $A$ to $B$, and $\left(B^{A}\right)_{\downarrow}$ denotes the space of all anti-monotone functions. Monotone and anti-monotone functions are closed under pointwise meet and join; thus, if $B$ is a lattice, then so are $[A \rightarrow B]_{\uparrow}$ and $[A \rightarrow B]_{\downarrow}$.

\subsection{Truth Domains and Sets}

A truth-domain $\mathcal{D}$ is a collection of elements $D$, referred to as truth values, together with a truth ordering $\sqsubseteq$ and a negation operator $\neg: D \rightarrow D$, such that $\mathcal{D}=(D, \sqsubseteq, \neg)$ is a De Morgan algebra. The truth ordering orders the elements based on their truth content; thus, $a \sqsubseteq b$ stands for " $a$ is less true than $b$ ". The meet $(\wedge)$ and join $(\vee)$ of the truth ordering are called conjunction and disjunction, respectively.

The best known truth domain is the classical boolean logic 2 with values true and false. Its truth ordering is shown in the Hasse diagram in Figure 1(a), with negation indicated in parentheses. Other examples include Belnap logic $\mathcal{B}$, shown in Figure 1(b), which extends boolean logic with two additional values: $m$ and $d$, to represent "unknown" and "inconsistent", respectively; and Fuzzy logic $\mathcal{F}$, shown in Figure 1(c). The truth values of $\mathcal{F}$ are formed by the set of all real numbers in the closed interval $[0,1]$, where 0 stands for false, 1 for true, and the remaining values stand for degrees of truth; furthermore, negation is defined as $\neg x \triangleq 1-x$, so that $\neg 0=1$ and $\neg 1=0$. 

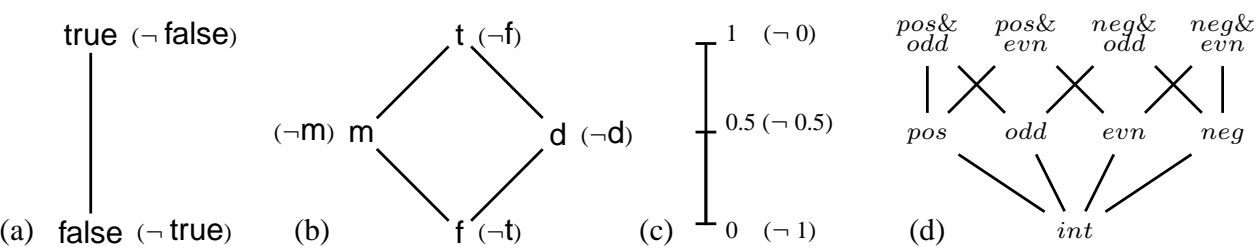

Fig. 1. (a)-(c) Truth domains: (a) 2-valued boolean logic, (b) Belnap logic, and (c) Fuzzy logic. (d) An abstract domain for $\mathbb{Z}$.

Given a collection of elements $U$, a set over $U$ is a function from $U$ to a truth domain. Thus, a boolean (or classical) set is a function from $U$ to 2, a Belnap set is a function from $U$ to $\mathcal{B}$, and a fuzzy set is a function from $U$ to $\mathcal{F}$. Set ordering and operations are defined by pointwise extensions. Let $S_{1}, S_{2}: U \rightarrow D$ be two sets. Then

$$
\begin{aligned}
S_{1} \subseteq S_{2} & \triangleq \forall x \cdot S_{1}(x) \sqsubseteq S_{2}(x) & & S_{1} \cup S_{2} \triangleq \lambda x \cdot S_{1}(x) \vee S_{2}(x) \\
S_{1} & \triangleq \lambda x \cdot \neg S_{1}(x) & & S_{1} \cap S_{2} \triangleq \lambda x \cdot S_{1}(x) \wedge S_{2}(x)
\end{aligned}
$$

\subsection{Modal $\mu$-Calculus}

In this section, we describe the modal $\mu$-calculus [20], or $L_{\mu}$.

Definition 1. Let $\mathrm{Var}$ be a set of variables and AP be a set of atomic propositions. The logic $L_{\mu}(A P)$ is the set of all formulas satisfying the grammar

$$
\varphi::=p|z| \neg \varphi|\varphi \wedge \varphi| \diamond \varphi \mid \mu z \cdot \varphi(z),
$$

where $p \in A P$, and $z \in$ Var. Furthermore, $z$ in $\mu z \cdot \varphi(z)$ must occur under the scope of an even number of negations in $\varphi(z)$.

Additionally, we define the following syntactic abbreviations:

$$
\varphi \vee \psi \triangleq \neg(\neg \varphi \wedge \neg \psi) \quad \varphi \Rightarrow \psi \triangleq \neg \varphi \vee \psi \quad \square \varphi \triangleq \neg \diamond \neg \varphi \quad \nu Z \cdot \varphi(Z) \triangleq \neg \mu Z \cdot \neg \varphi(\neg Z)
$$

The modal operator $\diamond$ is typically interpreted as "an existence of an immediate future". For example, " $p$ " means that $p$ holds now, " $\nabla p$ " means that there exists an immediate future where $p$ holds, and " $\square p$ " means that $p$ holds in all immediate futures. The quantifiers $\mu$ and $\nu$ stand for least and greatest fixpoint, respectively.

An occurrence of a variable $z$ in a formula $\varphi$ is bound if it appears in the scope of a $\mu$ quantifier and is free otherwise. For example, $z$ is free in $p \vee \diamond z$, and is bound in $\mu z \cdot p \vee \diamond z$. A formula $\varphi$ is closed if it does not contain any free variables.

A set-based interpretation of $L_{\mu}$ over a set domain $\mathcal{D}^{C}$ is a mapping $\|\cdot\|$ from closed $L_{\mu}$ formulas to $\mathcal{D}$-sets over $C$. The elements of $C$ are often called states, and $\|\varphi\|(c)=v$ is read as "the degree to which $\varphi$ is satisfied by (or, true in) a state $c$ is $v$ ".

An $L_{\mu}$ model is a structure $\mathcal{M}=\left(\mathcal{D}^{C},\left(p^{\mathcal{M}}\right)_{p \in A P}, \diamond^{\mathcal{M}}\right)$, where $\mathcal{D}^{C}$ is a set domain; for each $p \in A P, p^{\mathcal{M}}$ is in $\mathcal{D}^{C}$; and $\diamond^{\mathcal{M}}: \mathcal{D}^{C} \rightarrow \mathcal{D}^{C}$ is a $\subseteq$-monotone function. The set domain is called the universe of $\mathcal{M}$, and $p^{\mathcal{M}}$ and $\diamond^{\mathcal{M}}$ are interpretations of atomic propositions and the $\diamond$ operator, respectively. A model $\mathcal{M}$ gives rise to an $L_{\mu}$ interpretation $\|\cdot\|^{\mathcal{M}}$.

The interpretation $\|\varphi\|_{\sigma}^{\mathcal{M}}$ is defined inductively on the structure of the formula $\varphi$, where $\sigma: \operatorname{Var} \rightarrow \mathcal{D}^{C}$ is an object assignment for free variables:

$$
\begin{array}{ll}
\|p\|_{\sigma}^{\mathcal{M}} \triangleq p^{\mathcal{M}} & \|z\|_{\sigma}^{\mathcal{M}} \triangleq \sigma(z) \\
\|\varphi \wedge \psi\|_{\sigma}^{\mathcal{M}} \triangleq\|\varphi\|_{\sigma}^{\mathcal{M}} \cap\|\psi\|_{\sigma}^{\mathcal{M}} & \|\neg \varphi\|_{\sigma}^{\mathcal{M}} \triangleq \overline{\|\varphi\|_{\sigma}^{\mathcal{M}}} \\
\|\mu x \cdot \varphi\|_{\sigma}^{\mathcal{M}} \triangleq \operatorname{lfp} \subseteq\left(\lambda S \cdot\|\varphi\|_{\sigma[x \mapsto S]}^{\mathcal{M}}\right) & \|\nabla \varphi\|_{\sigma}^{\mathcal{M}} \triangleq \diamond^{\mathcal{M}}\left(\|\varphi\|_{\sigma}^{\mathcal{M}}\right)
\end{array}
$$


where $\operatorname{lfp} \subseteq f$ is the $\subseteq$-least fixpoint of $f$. For a closed $L_{\mu}$ formula $\varphi,\|\varphi\|_{\sigma}^{\mathcal{M}}=\|\varphi\|_{\sigma^{\prime}}^{\mathcal{M}}$ for any $\sigma$ and $\sigma^{\prime}$. Thus, we write $\|\varphi\|^{\mathcal{M}}$ for that value, and define it to be the modelbased interpretation of $\varphi$.

Formulas of $L_{\mu}$ are often interpreted over Kripke structures. A Kripke structure is a tuple $\mathcal{K}=(A P, C, \mathcal{D}, I, R)$, where $A P$ is a collection of atomic propositions, $C$ is a collection of elements (called states), $\mathcal{D}$ is a truth domain, $I: A P \rightarrow \mathcal{D}^{C}$ is a mapping from atomic propositions to sets over $C$, and $R: C \rightarrow \mathcal{D}^{C}$ is a transition function mapping each state to a set of its successors. For a transition function $R$, we define a pre-image operator pre $[R]: \mathcal{D}^{C} \rightarrow \mathcal{D}^{C}$ and its dual pre $[R]$ as:

$$
\operatorname{pre}[R](Q)(s) \triangleq \vee_{t \in C}(R(s) \cap Q)(t) \quad \text { pre }[R](Q) \triangleq \overline{\operatorname{pre}[R](\bar{Q})}
$$

Intuitively, $\operatorname{pre}[R](Q)(s)$ is a degree to which the set $R(s)$ of successors of $s$ has a non-empty intersection with $Q$. A Kripke structure $\mathcal{K}=(A P, C, \mathcal{D}, I, R)$ gives rise to

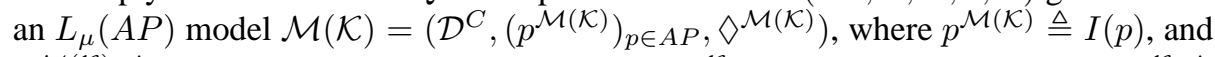

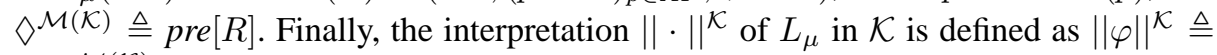
$\|\varphi\|^{\mathcal{M}(\mathcal{K})}$.

\subsection{Abstract Interpretation}

The framework of Abstract Interpretation (AI) provides a collection of tools for systematic design and analysis of semantic approximations [7]. The framework is very flexible and can be applied in various ways. Below, we give a brief overview of AI, summarizing the results used in our work.

Basics of Abstract Interpretation. Inputs to an AI framework are collections of concrete elements $C$ and abstract elements $A$, called a concrete and an abstract domain, respectively. A notion of approximation, or abstraction, is formalized by a soundness relation $\rho \subseteq A \times C$, where $a \rho_{c}$ is read as " $a \rho$-approximates $c$ ".

A concretization function $\gamma: A \rightarrow 2^{C}$ maps each abstract element to a set of concrete elements corresponding to it: $\gamma(a) \triangleq\{c \mid a \rho c\}$. An abstract element $a$ is called consistent if $\gamma(a) \neq \emptyset$; otherwise, we say $a$ is inconsistent. The elements of $A$ can be thought of as properties, such as "positive" or "odd", and $\gamma(a)$ as a collection of concrete elements satisfying $a$. The concretization $\gamma$ induces an approximation ordering $\preceq_{\rho}$ on $A$ such that $a \preceq_{\rho} b \Leftrightarrow \gamma(a) \supseteq \gamma(b)$.

Intuitively, $a \preceq_{\rho} b$ means that $a$ approximates more concrete elements than $b$; therefore, $a$ is less informative, or equivalently, less precise than $b$. When viewed as a property, $a$ is weaker than $b$. For example, knowing that an element is "positive" is less informative than knowing that it is both "positive" and "odd".

In this paper, an abstract domain $A$ is equipped with an information ordering $\preceq_{A}$ such that $\left(A, \preceq_{A}\right)$ is a lattice and $a \preceq_{A} b \Rightarrow a \preceq_{\rho} b$. Thus, we can study properties of an abstract domain independently of any particular soundness relation. Furthermore, we assume that $A$ satisfies "the existence of a best approximation" [7], that is:

$$
\forall c \in C \cdot \exists a \in A \cdot\left(a \rho_{c} \wedge \forall a^{\prime} \in A \cdot a^{\prime} \rho_{c} \Rightarrow \gamma\left(a^{\prime}\right) \supseteq \gamma(a)\right)
$$

and use $\alpha: C \rightarrow A$ to denote an abstraction function that maps each concrete element to its best approximation. Note that for a given $c, A$ may have several best approximations; thus, $\alpha$ is not uniquely defined. In such cases, it is convenient to 
use the $\preceq_{A}$-largest $\alpha$, so that $\rho$ and $\gamma$ can be expressed as $a \rho c \Leftrightarrow a \preceq_{A} \alpha(c)$ and $c \in \gamma(a) \Leftrightarrow a \preceq A \alpha(c)$, respectively.

A lower bound with respect to $\preceq_{\rho}$ is called widening and is denoted by $\nabla$. Intuitively, for a set $Q \subseteq A, \nabla Q$ is an abstract element representing the information common to all elements of $Q$, i.e., $\gamma(\nabla Q) \supseteq \cup_{q \in Q} \gamma(q)$. In particular, the greatest lower bound $\Pi_{A}$ of $\preceq_{A}$ is a widening. A widening $\nabla$ is info-preserving if for any $Q$ containing no inconsistent elements, $\nabla Q$ is the best representation of information common to all elements of $Q$, i.e., $\forall a \in A \cdot \gamma(a) \supseteq \cup_{q \in Q} \gamma(q) \Rightarrow \gamma(a) \supseteq \gamma(\nabla Q)$.

Abstract domains $\left(A_{1}, \preceq_{1}\right)$ and $\left(A_{2}, \preceq_{2}\right)$ are informationally equivalent if they represent the same degrees of information, that is, $\forall a_{1} \in A_{1} \cdot \exists a_{2} \in A_{2} \cdot \gamma_{1}\left(a_{1}\right)=\gamma_{2}\left(a_{2}\right)$ and $\forall a_{2} \in A_{2} \cdot \exists a_{1} \in A_{1} \cdot \gamma_{1}\left(a_{1}\right)=\gamma_{2}\left(a_{2}\right)$.

For examples in this paper, we use the set of integers $\mathbb{Z}$ as a concrete domain, and the domain $\mathbb{A}$, shown in Figure 1(d), as the abstract domain. The soundness relation $\rho_{e} \subseteq$ $\mathbb{A} \times \mathbb{Z}$ is self-explanatory, e.g., 2 is $\rho_{e}$-approximated by pos\&evn, evn, pos, and int, where pos\&evn is its best abstract approximation. Similarly, $\gamma_{e}(e v n)$ is the set EVEN of all even numbers, $\gamma_{e}(n e g)$ is the set $N E G$ of all negative numbers, $\gamma_{e}(n e g \& e v n)$ is $N E G \cap E V E N$, etc.

Functional Abstraction. In practice, it is common to synthesize abstractions of complex structures using abstractions of their parts. A particular application is abstraction of functions, or functional abstraction [7].

Let $\mathcal{A}=A_{1} \rightarrow A_{2}$ and $\mathcal{C}=C_{1} \rightarrow C_{2}$ be collections of abstract and concrete functions, where $A_{1}$ and $A_{2}$ are abstract domains approximating $C_{1}$ and $C_{2}$, respectively. A soundness relation $\rho_{f} \subseteq \mathcal{A} \times \mathcal{C}$ is functional if $g \rho_{f}$-approximates $f$ iff $g$ preserves soundness of $f$. Formally, $\rho_{f}$ satisfies

$$
g \rho_{f} f \Leftrightarrow \forall a_{1} \in A_{1} \cdot \forall c_{1} \in \gamma_{1}\left(a_{1}\right) \cdot g\left(a_{1}\right) \rho_{2} f\left(c_{1}\right) \quad \text { (functional soundness) }
$$

Let $\nabla$ be a widening operator of $A_{2}$, and $\alpha_{\nabla}: \mathcal{C} \rightarrow \mathcal{A}$ be defined as

$$
\alpha_{\nabla}(f)(a) \triangleq \nabla_{c \in \gamma_{1}(a)} \alpha_{2}(f(c)) \quad \text { (functional abstraction) }
$$

Then $\alpha_{\nabla}(f)$ is a $\rho_{f}$-approximation of $f$, and its precision is determined by the precision of the widening operator used.

Theorem 1. [7] Let $\mathcal{A}, \mathcal{C}, \rho_{f}$, and $\alpha_{\nabla}$ be as above. If $\nabla$ is info-preserving, then $\alpha_{\nabla}(f)$ is the best $\rho_{f}$-approximation of $f$.

One of the main results of $\mathrm{AI}$ is that $\alpha_{\nabla}$ preserves fixpoints:

Theorem 2. [7] Let $\left(C, \sqsubseteq_{C}\right)$ be a lattice, $f:[C \rightarrow C]_{\uparrow}$ be a monotone function, and $\left(A, \sqsubseteq_{A}\right)$ be a lattice approximating $C$ via $\rho_{C}$. If the join operator $\vee_{A}$ of $A$ preserves soundness, i.e., $\left(\alpha_{C}\left(c_{1}\right) \vee_{A} \alpha_{C}\left(c_{2}\right)\right) \preceq_{A} \alpha_{C}\left(c_{1} \vee_{C} c_{2}\right)$, then the least fixpoint of $\alpha_{\nabla}(f)$ $\rho_{C}$-approximates the least fixpoint of $f: \operatorname{lfp}^{{ }^{A}} \alpha_{\nabla}(f) \rho_{C}$ lfp ${ }^{{ }_{C}} f$.

Functional Abstraction and Monotone Functions. Let $\mathcal{A}=\left[A_{1} \rightarrow A_{2}\right]$ be as above, and assume that $A_{1}$ and $A_{2}$ are equipped with information orderings $\preceq_{1}$ and $\preceq_{2}$, respectively. Then the set $\mathcal{A}_{\uparrow}=\left[A_{1} \rightarrow A_{2}\right]_{\uparrow}$ of $\preceq$-monotone functions is informationally equivalent to $\mathcal{A}$.Furthermore, if $\nabla$ is an info-preserving widening of $A_{2}$, then its pointwise extension to functions is also an info-preserving widening of $\mathcal{A}_{\uparrow}$ [25]. Therefore, we always restrict the abstract domain of functional abstraction to $\preceq$-monotone functions. 


\section{Abstract Sets}

Sets play the role of basic blocks in the definition of $L_{\mu}$ semantics. In this section, we develop an abstraction of sets that preserves all set operations, including set complement. This abstraction gives us the necessary tools for abstracting $L_{\mu}$ models, which we do in Section 4. But it is independent of $L_{\mu}$ and can be used anywhere abstract sets are required.

We assume that $C$ and $A$ are a concrete and an abstract domain, respectively, related by a soundness relation $\rho_{e}$ and an abstraction function $\alpha_{e}$. We aim to lift $\rho_{e}$ to a soundness relation $\rho_{s}$ between concrete sets, i.e., functions from $C$ into a fixed truth domain $\mathcal{D}$, and abstract sets, i.e., functions from $A$ into some truth domain $\mathcal{B}$ (potentially different from $\mathcal{D}$ ). The goal of $\rho_{s}$ is to preserve set membership: that is, if $S_{\alpha}$ $\rho_{s}$-approximates $S$, then if $a \in A \rho_{e}$-approximates $c, S_{\alpha}(a)$ must approximate $S(c)$. As always, we also want to know when $S_{\alpha}$ is a best approximation of a given set $S$.

We view sets as functions, so it is natural to express $\rho_{s}$ as a functional abstraction. For this, we must first identify the notion of an abstract truth domain $\mathcal{B}$ and settle on the meaning of "approximating truth values".

\subsection{Bilattices as Abstract Truth Domains}

Intuitively, an abstract truth-domain $\mathcal{B}$ is a truth-domain and, therefore, has a truth ordering and a negation. It is also an abstract domain and needs an information ordering. Furthermore, truth operations should not interfere with the information ordering. For example, if $a$ and $b$ are in $\mathcal{B}$ and $a$ is less informative than $b$, then negation of $a(\neg a)$ must be less informative than $\neg b$.

A structure that captures our intuition is that of a bilattice, which has been introduced by Ginsberg [15] to enable reasoning with partiality and inconsistency. Here, we briefly describe distributive bilattices.

Definition 2. [15] A distributive bilattice is a structure $\mathcal{B}=(B, \preceq, \sqsubseteq, \neg)$ such that: (1) $\mathcal{B}_{i}=(B, \preceq)$ is a lattice and $\mathcal{B}_{t}=(B, \sqsubseteq, \neg)$ is a De Morgan algebra; (2) meet ( $\left.\square\right)$ and join $(\sqcup)$ of $\mathcal{B}_{i}$, and meet $(\wedge)$ and join $(\vee)$ of $\mathcal{B}_{t}$ are monotone with respect to both $\preceq$ and $\sqsubseteq$; (3) all meets and joins distribute over each other; and (4) negation ( $\neg$ ) is $\preceq$-monotone.

The ordering $\preceq$ ranks elements of $\mathcal{B}$ with respect to information, and $\sqsubseteq$ ranks them with respect to truth. Operations $\wedge$ and $\vee$ of $\mathcal{B}_{t}$ are called conjunction and disjunction. In the spirit of AI, we refer to $\sqcap$ and $\sqcup$ as widening and narrowing, respectively.

De Morgan algebras have a natural connection to bilattices.

Theorem 3. $[15,14]$. Let $\mathcal{D}=(D, \leq,-)$ be a De Morgan algebra, and $\mathcal{B}(\mathcal{D})$ be a structure $(D \times D, \preceq, \sqsubseteq, \neg)$ such that

$$
\langle a, b\rangle \preceq\langle c, d\rangle \triangleq a \leq c \wedge b \leq d \quad\langle a, b\rangle \sqsubseteq\langle c, d\rangle \triangleq a \leq c \wedge d \leq b \quad \neg\langle a, b\rangle \triangleq\langle b, a\rangle
$$

Then, $\mathcal{B}(\mathcal{D})$ is a distributive bilattice. Furthermore, every distributive bilattice is isomorphic to $\mathcal{B}(\mathcal{D})$ for some De Morgan algebra $\mathcal{D}$.

For a truth-domain $\mathcal{D}$, an element $\langle x, y\rangle$ of $\mathcal{B}(\mathcal{D})$ is interpreted as a truth value whose degree of truth is $x$ and degree of falsity is $y$. For example, $\mathcal{B}(\mathbf{2})$ consists of four elements: $\langle t, f\rangle$ representing true - maximal degree of truth and minimal degree of 
falsity, $\langle f, t\rangle$ representing false, $\langle f, f\rangle$ representing lack of knowledge - minimal degree of both truth and falsity, and $\langle t, t\rangle$ representing an inconsistency (or disagreement) maximal degree of both truth and falsity. It is easy to verify that $\mathcal{B}(\mathbf{2})$ is exactly Belnap logic shown in Figure 1(b). For convenience, we introduce projections $\pi_{\mathrm{t}}$ and $\pi_{\mathrm{f}}$ defined as $\pi_{\mathrm{t}}(\langle x, y\rangle) \triangleq x$ and $\pi_{\mathrm{f}}(\langle x, y\rangle) \triangleq y$.

Guided by the above intuition, we say that $\mathcal{B}(\mathcal{D})$ is an abstract truth-domain corresponding to a truth domain $\mathcal{D}$. Intuitively, $\langle x, y\rangle \in \mathcal{B}(\mathcal{D})$ approximates $c \in \mathcal{D}$ if $x$ is no more true than $c$, and $y$ is no more false than $c$. In particular, $\langle c,-c\rangle$ is the best approximation of $c$. Formally, this is captured by an abstraction function $\alpha_{t}(c) \triangleq\langle c,-c\rangle$, and a soundness relation $\rho_{t} \triangleq\left\{(a, c) \mid a \preceq \alpha_{t}(c)\right\}$.

It is easy to verify that truth operations of $\mathcal{B}(\mathcal{D})$, including negation, preserve soundness. That is, if $a_{1} \preceq \alpha_{t}\left(c_{1}\right)$ and $a_{2} \preceq \alpha_{t}\left(c_{2}\right)$, then $a_{1} \wedge a_{2} \preceq \alpha_{t}\left(c_{1} \wedge c_{2}\right), a_{1} \vee a_{2} \preceq$ $\alpha_{t}\left(c_{1} \vee c_{2}\right)$, and $\neg a_{1} \preceq \alpha_{t}\left(\neg c_{1}\right)$. Furthermore, $\sqcap$ is an info-preserving widening.

\subsection{Set Abstraction}

We now formally define the soundness relation $\rho_{s}$ between concrete $(C \rightarrow \mathcal{D})$ and abstract $(A \rightarrow \mathcal{B}(\mathcal{D}))$ sets as:

$$
S_{\alpha} \rho_{s} S \triangleq \forall a \in A \cdot \forall c \in \gamma_{e}(a) \cdot S_{\alpha}(a) \preceq \alpha_{t}(S(c)) \quad \text { (set soundness) }
$$

The soundness relation $\rho_{s}$ is functional, and the corresponding abstraction function $\alpha_{s}$ follows immediately from Theorem 1 :

$$
\alpha_{s}(S)(a) \triangleq \sqcap_{c \in \gamma_{e}(a)} \alpha_{t}(S(c)) \quad \text { (set abstraction) }
$$

Note that $\alpha_{s}(S)(a)=\langle x, y\rangle$ means that the elements in $\gamma_{e}(a)$ belong to $S$ with a truth degree of at least $x$, and to $\bar{S}$ with a truth degree of at least $y$. In particular, if $S$ is a boolean set, then $\alpha_{s}(S)$ is a Belnap set; $\alpha_{s}(S)(a)$ is t iff $\gamma_{e}(a)$ is contained in $S, \mathrm{f}$ iff $\gamma_{e}(a)$ is contained in $\bar{S}, \mathrm{~m}$ iff $\gamma_{e}(a)$ is not contained in either $S$ or $\bar{S}$, and d iff $\gamma_{e}(a)$ is contained in both $S$ and $\bar{S}$.

For example, an abstraction $\alpha_{s}(E V E N)$ of a boolean set $E V E N \in \mathbf{2}^{\mathbb{Z}}$ is

$$
\alpha_{s}(E V E N)(a) \triangleq \begin{cases}\mathrm{t} & \text { if } \gamma_{e}(a) \subseteq E V E N \\ \mathrm{f} & \text { if } \gamma_{e}(a) \subseteq O D D \\ \mathrm{~m} & \text { otherwise }\end{cases}
$$

Note a difference between an abstract element evn and an abstract set $\alpha_{s}(E V E N)$. The former represents a property of being an even number, and $\gamma_{e}($ evn $)=E V E N$ is the set of all numbers having this property. On the other hand, $\alpha_{s}(E V E N)$ represents a set that contains all even and no odd numbers; hence, $\gamma_{s}\left(\alpha_{s}(E V E N)\right)=\{E V E N\}$ is a singleton containing the only set satisfying these conditions.

Recall that the set operations of $\mathcal{B}(\mathcal{D})^{A}$ are pointwise extensions of the corresponding operations of $\mathcal{B}(\mathcal{D})$; therefore, they preserve soundness. For example, if $S_{\alpha} \rho_{s^{-}}$approximates $S$, then $\overline{S_{\alpha}} \rho_{s}$-approximates $\bar{S}$, etc.

Finally, since $\rho_{s}$ is functional, following the discussion in Section 2.4, we restrict the domain of abstract sets to $\preceq$-monotone functions, i.e., to $\mathcal{B}(\mathcal{D})_{\uparrow}^{A}$. Note that abstract set operations preserve $\preceq$-monotonicity and do not interfere with this restriction. This gives us with a abstract domain for sets that (a) preserves all set operations and (b) has an info-preserving widening. We use elements of this abstract domain as basic blocks for designing $L_{\mu}$-preserving abstractions in the next section. 

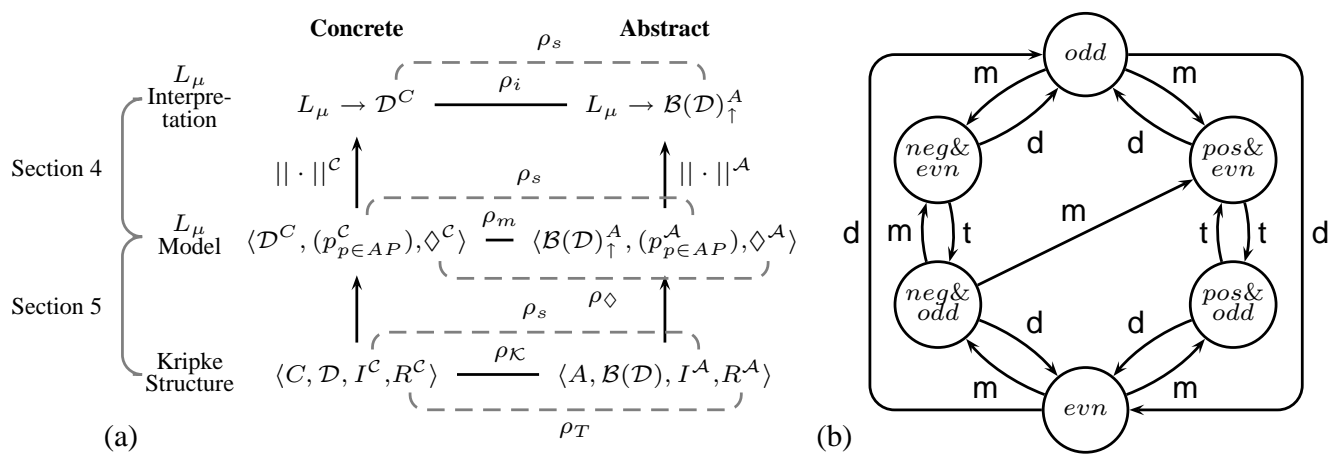

Fig. 2. (a) Abstracting $L_{\mu}$ : the top row summarizes soundness relations for abstracting $L \mu$ interpretations; the middle one $-L_{\mu}$ models, i.e., interpretions of atomic propositions and $\diamond$ relation; the bottom one $-L_{\mu}$-preserving abstractions of Kripke structures. (b) A fragment of the abstraction $\alpha_{T}\left(R_{1}\right)$, where $R_{1}(x)=x+1$.

\section{Abstract Interpretation for Modal $\mu$-Calculus}

In this section, we develop an abstraction of $L_{\mu}$ models that is sound w.r.t. satisfaction and refutation of all $L_{\mu}$ formulas, i.e., if an $L_{\mu}$ formula is satisfied (refuted) by the abstract model, it is satisfied (refuted) by the concrete one. We start by formalizing the notion of $L_{\mu}$-preserving approximation in the language of AI, and then systematically extend it to the desired abstraction. The top half of the diagram in Figure 2(a) illustrates the structures and relations discussed in this section, where solid lines represent relations between structures, and dashed those between their components.

We assume that $C$ is a collection of concrete elements, called states, and $\mathcal{D}$ is a truth domain. Recall from Section 2.3 that an interpretation of $L_{\mu}\|\cdot\|$ over a set domain $\mathcal{D}^{C}$ maps each closed $L_{\mu}$ formula to a $\mathcal{D}$-set over $C$, where $\|\varphi\|(c)$ is the degree to which a formula $\varphi$ is true in a state $c$.

Let $A$ be an abstract domain approximating $C$ via a soundness relation $\rho_{e}$, and $\mathcal{B}(\mathcal{D})$ be an abstract truth domain approximating $\mathcal{D}$ via a soundness relation $\rho_{t}$ as defined in Section 3.1. Furthermore, let $\|\cdot\|_{\alpha}$ be an interpretation of $L_{\mu}$ formulas as $\mathcal{B}(\mathcal{D})$-sets over $A$.

A natural way to extend the soundness relation $\rho_{e}$ from states to $L_{\mu}$ interpretations is to say that $\|\cdot\|_{\alpha}$ approximates $\|\cdot\|$ if for every $L_{\mu}$ formula $\varphi$ and every abstract state $a \in A,\|\varphi\|_{\alpha}(a)$ approximates the degree to which $\|\varphi\|$ is true for every concrete state $c$ corresponding to $a$. We denote this soundness relation by $\rho_{i}$ and formalize it using the set soundness relation $\rho_{s}$, defined in Section 3.2, as

$$
\left.\|\cdot\|_{\alpha} \rho_{i}\|\cdot\| \triangleq \forall \varphi \in L_{\mu} \cdot\|\varphi\|_{\alpha} \rho_{s}\|\varphi\| \quad \text { ( } L_{\mu} \text { soundness }\right)
$$

In this paper, we are only interested in the model-based interpretations of $L_{\mu}$. A natural way to extend $\rho_{i}$ to models is to say that a concrete model $\mathcal{C}$ is approximated by an abstract model $\mathcal{A}$ if the corresponding $L_{\mu}$ interpretation $\|\cdot\|^{\mathcal{C}}$ is approximated by $\|\cdot\|^{\mathcal{A}}$. Formally, we define a model soundness relation $\rho_{m}$ as

$$
\mathcal{A} \rho_{m} \mathcal{C} \triangleq\|\cdot\|^{\mathcal{A}} \rho_{i}\|\cdot\|^{\mathcal{C}} \quad \text { (model soundness) }
$$

In the rest of this section, we employ the AI framework to construct an abstract model $\mathcal{A}$ that is a best $\rho_{m}$-approximation of a given concrete model $\mathcal{C}$. As discussed in Section 3, we restrict the universe of $\mathcal{A}$ to $\preceq$-monotone functions from $A$ to $\mathcal{B}(\mathcal{D})$. 
We first outline the steps involved: (1) define a soundness relation $\rho_{\diamond}$ between interpretations of the $\diamond$ operator and derive the corresponding abstraction function $\alpha_{\diamond}$; (2) show that an abstract model $\mathcal{A}=\left(\mathcal{B}(\mathcal{D})_{\uparrow}^{A},\left(p^{\mathcal{A}}\right)_{p \in A P}, \diamond^{\mathcal{A}}\right) \rho_{m}$-approximates a concrete model $\mathcal{C}=\left(\mathcal{D}^{C},\left(p^{\mathcal{C}}\right)_{p \in A P}, \diamond^{\mathcal{C}}\right)$ if for each $p \in A P, p^{\mathcal{A}} \rho_{s}$-approximates $p^{\mathcal{C}}$, and $\nabla^{\mathcal{A}} \rho_{\diamond}$-approximates $\nabla^{\mathcal{C}}$; (3) conclude that the best approximation of $\mathcal{C}$ is given by $\alpha_{m}(\mathcal{C}) \triangleq\left(\mathcal{B}(\mathcal{D})_{\uparrow}^{A},\left(\alpha_{s}\left(p^{\mathcal{C}}\right)\right)_{p \in A P}, \alpha_{\diamond}\left(\diamond^{\mathcal{C}}\right)\right)$.

Step 1. For a given $L_{\mu}$-model, an interpretation of modal formulas, i.e. formulas with $\diamond$ but no fixpoint quantifiers, is determined by the model's interpretation of the $\diamond$ operator. Thus, we define $\rho_{\diamond}$ as follows:

$$
\diamond^{\mathcal{A}} \rho_{\diamond} \diamond^{\mathcal{C}} \triangleq \forall X \in \mathcal{B}(\mathcal{D})_{\uparrow}^{A} \cdot \forall Y \in \gamma_{s}(X) \cdot \diamond^{\mathcal{A}}(X) \rho_{s} \diamond^{\mathcal{C}}(Y) \quad(\diamond \text {-soundness })
$$

Following Theorem 1, its corresponding abstraction function $\alpha_{\diamond}$ is defined as

$$
\alpha_{\diamond}\left(\diamond^{\mathcal{C}}\right)(X) \triangleq \sqcap_{Y \in \gamma_{s}(X)} \alpha_{s}\left(\diamond^{\mathcal{C}}(Y)\right) \quad(\diamond \text {-abstraction })
$$

Step 2. To show that an abstract model $\mathcal{A} \rho_{m}$-approximates a concrete model $\mathcal{C}$ if each component of $\mathcal{A}$ approximates the corresponding counterpart of $\mathcal{C}$, we need to show that for any formula $\varphi,\|\varphi\|^{\mathcal{A}} \rho_{s}$-approximates $\|\varphi\|^{\mathcal{C}}$.

Theorem 4. Let $\mathcal{C}=\left(\mathcal{D}^{C},\left(p^{\mathcal{C}}\right)_{p \in A P}, \diamond^{\mathcal{C}}\right)$ be a concrete model, $\mathcal{A}=\left(\mathcal{B}(\mathcal{D})^{A},\left(p^{\mathcal{A}}\right)_{p \in A P}\right.$, $\left.\diamond^{\mathcal{A}}\right)$ be an abstract model such that $A$ approximates $C$ via a soundness relation $\rho_{e}$. Then, $\mathcal{A} \rho_{m} \mathcal{C} \Leftarrow \forall p \in A P \cdot p^{\mathcal{A}} \rho_{s} p^{\mathcal{C}} \wedge \diamond^{\mathcal{A}} \rho_{\diamond} \diamond^{\mathcal{C}}$.

The theorem is proved by structural induction on $\varphi$, using Theorem 2 for cases where $\varphi$ contains a fixpoint quantifier.

Step 3. Finally, we define an abstraction function $\alpha_{m}$ that maps each concrete model to its best abstract approximation:

$$
\alpha_{m}(\mathcal{C}) \triangleq\left(\mathcal{B}(\mathcal{D})_{\uparrow}^{A},\left(\alpha_{s}\left(p^{\mathcal{C}}\right)\right)_{p \in A P}, \alpha_{\diamond}\left(\diamond^{\mathcal{C}}\right)\right) \quad \text { (model abstraction) }
$$

For example, consider a concrete boolean model $\mathcal{C}=\left(\mathbf{2}^{\mathbb{Z}}, p^{\mathcal{C}}, \diamond^{\mathcal{C}}\right)$, where $p^{\mathcal{C}}=$ EVEN and $\diamond^{\mathcal{C}}=\lambda S \cdot\{y \mid y+1 \in S\}$. Then, $\diamond p$ is interpreted in $\mathcal{C}$ as $\|\nabla p\|^{\mathcal{C}}=$ $\diamond^{\mathcal{C}}(E V E N)=O D D$, and in the abstraction of $\mathcal{C}$ as $\|\diamond p\|^{\alpha_{m}(\mathcal{C})}=\alpha_{\diamond}\left(\diamond^{\mathcal{C}}\right)\left(\alpha_{s}(E V E N)\right)=$ $\alpha_{s}(O D D)$.

The resulting abstraction function $\alpha_{m}$ allows us to abstract $L_{\mu}$ models, obtaining abstractions which are both sound and precise. However, $\alpha_{m}$ depends on an interpretation of $\diamond$ modality, which we left unspecified. We study this subject below.

\section{Abstraction of Kripke Structures}

In practice, the $\diamond$ modality is often interpreted using a Kripke structure. In this section, we are interested in conditions under which a Kripke structure over an abstract statespace (i.e., an abstract Kripke structure) is a best approximation of a given concrete one. We show that the framework of AI provides an elegant and almost mechanical way to answer this question.

Approximating Kripke Structures. Below, we aim to extend the soundness relation $\rho_{m}$ between models to a soundness relation $\rho_{\mathcal{K}}$ between Kripke structures, and derive a corresponding abstraction function $\alpha_{\mathcal{K}}$.

Throughout this section, we assume that $\mathcal{C}=\left(C, \mathcal{D}, I^{\mathcal{C}}, R^{\mathcal{C}}\right)$ is a concrete Kripke structure over concrete states $C$ and a truth domain $\mathcal{D}$, and $\mathcal{A}=\left(A, \mathcal{B}(\mathcal{D}), I^{\mathcal{A}}, R^{\mathcal{A}}\right)$ 


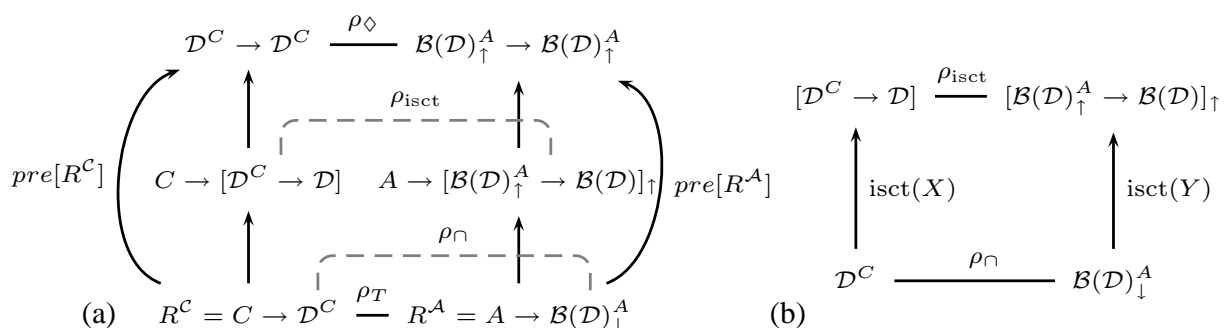

Fig. 3. (a) Soundness relations between $\diamond$ modality and transition function; (b) Detail of (a): relations $\rho_{\text {isct }}$ and $\rho_{\cap}$.

is an abstract Kripke structure, where $A$ is an abstract domain related to $C$ via $\rho_{e}$, and $\mathcal{B}(\mathcal{D})$ is an abstract truth domain related to $\mathcal{D}$ via $\rho_{t}$.

The soundness relation $\rho_{\mathcal{K}}$ on Kripke structures is defined as a restriction of the model soundness relation $\rho_{m}$ (see Figure 2(a)):

$$
\mathcal{A} \rho_{\mathcal{K}} \mathcal{C} \triangleq \mathcal{M}(\mathcal{A}) \rho_{m} \mathcal{M}(\mathcal{C})
$$

(Kripke soundness)

By Theorem 4, $\rho_{\mathcal{K}}$ is decomposed over the components of the Kripke structure:

$$
\mathcal{A} \rho_{\mathcal{K}} \mathcal{C} \Leftarrow\left(\forall p \in A P \cdot I^{\mathcal{A}}(p) \rho_{s} I^{\mathcal{C}}(p)\right) \wedge R^{\mathcal{A}} \rho_{T} R^{\mathcal{C}}
$$

where the relation $\rho_{T}$ between transition functions is defined as:

$$
R^{\mathcal{A}} \rho_{T} R^{\mathcal{C}} \triangleq \operatorname{pre}\left[R^{\mathcal{A}}\right] \rho_{\diamond} \operatorname{pre}\left[R^{\mathcal{C}}\right] \quad \text { (transition soundness) }
$$

The abstraction function $\alpha_{s}$ corresponding to $\rho_{s}$ has already been defined in Section 3.2. Thus, the only missing ingredient for defining $\alpha_{\mathcal{K}}$ is the transition abstraction $\alpha_{T}$. Unfortunately, the soundness relation $\rho_{T}$ is not functional; making Theorem 1 not applicable. However, we show below that $\rho_{T}$ can be easily made functional. We begin by introducing an intersection operator isct: isct $(X)(S) \triangleq \vee_{t}(X \cap S)(t)$ which allows us to express the pre-image of a transition function $R$ as pre $[R](Q)=\lambda s \cdot \operatorname{isct}(R(s))(Q)$. We then define a functional soundness relation $\rho_{\text {isct }}$ (see Figure 3(a)):

$$
\operatorname{isct}(X) \rho_{\text {isct }} \operatorname{isct}(Y) \triangleq \forall S \in \mathcal{B}(\mathcal{D})_{\uparrow}^{A} \cdot \forall Q \in \gamma_{s}(S) \cdot \operatorname{isct}(X)(S) \rho_{t} \operatorname{isct}(Y)(Q)
$$

Noticing that $\operatorname{isct}(X)$ is determined by a set $X$, we extend $\rho_{\text {isct }}$ to a soundness relation $\rho_{\cap}$ between sets (see Figure 3(b)):

$$
X \rho_{\cap} Y \triangleq \operatorname{isct}(X) \rho_{\text {isct }} \operatorname{isct}(Y)
$$

(successor soundness)

Finally, $\rho_{T}$ is made functional:

$$
\begin{aligned}
R^{\mathcal{A}} \rho_{T} R^{\mathcal{C}} & \Leftrightarrow \operatorname{pre}\left[R^{\mathcal{A}}\right] \rho_{\diamond} \operatorname{pre}\left[R^{\mathcal{C}}\right] \\
& \Leftrightarrow \forall a \in A \cdot \forall c \in \gamma_{e}(s) \cdot \operatorname{isct}\left(R^{\mathcal{A}}(a)\right) \rho_{\text {isst }} \operatorname{isct}\left(R^{\mathcal{C}}(c)\right) \\
& \Leftrightarrow \forall a \in A \cdot \forall c \in \gamma_{e}(s) \cdot R^{\mathcal{A}}(a) \rho_{\cap} R^{\mathcal{C}}(c)
\end{aligned}
$$

However, $\rho_{\cap}$ is still not functional! Thus, before applying Theorem 1 to construct $\alpha_{T}$, we need to construct the abstraction function $\alpha_{\cap}$ directly, i.e., without using Theorem 1 . We do so below.

Abstraction of Intersection. Intuitively, the ideal abstraction $\alpha_{\cap}$ is such that the diagram in Figure 3(b) commutes. That is, $\alpha_{\cap}(X)=Y$ implies that $\alpha_{\text {isct }}(\operatorname{isct}(X))=$ isct $(Y)$. Note that $\rho_{\text {isct }}$ is functional, thus the definition of $\alpha_{\text {isct }}(\operatorname{isct}(X))$ follows from Theorem 1. Following a standard technique of AI, we proceed to reorganize this definition until the emergence of conditions under which $Y \in \mathcal{B}(\mathcal{D})^{A}$ is the best $\rho_{\cap}$ abstraction of $X$. This derivation is simple but long, and is omitted from the paper. For details, please see full version of this paper [19]. Here, we only show the final result. 
Theorem 5. Let $C$ and $\left(A, \preceq_{A}\right)$ be a concrete and an abstract domain related by $\rho_{e}$, $\mathcal{D}$ and $\mathcal{B}(\mathcal{D})$ be truth-domains related by $\rho_{t}$, and for $X \in \mathcal{D}^{A}$, let $\alpha_{\cap}$ be defined as

$$
\alpha_{\cap}(X)(a) \triangleq\left\langle\vee_{c \in \gamma_{e}(a)} X(c), \wedge_{c \in \tilde{\gamma}_{e}(a)} \neg X(c)\right\rangle,
$$

where $\tilde{\gamma}_{e}(a) \triangleq\left\{c \in C \mid \alpha_{e}(c) \preceq_{A}\right.$ a $\}$ is a dual-conretization function. Then, $\alpha_{\text {isct }}(\operatorname{isct}(X))=\operatorname{isct}\left(\alpha_{\cap}(X)\right)$.

To construct $\alpha_{T}$ using Theorem 1, we need an info-preserving widening. The widening $\sqcap$ on $\mathcal{B}(D)^{A}$ - the pointwise extension of $\sqcap$ of $\mathcal{B}(\mathcal{D})$ - is not info-preserving in general. Instead, we restrict the abstract domain to the $\preceq$-antimonotone functions, i.e., to $\mathcal{B}(\mathcal{D})_{\downarrow}^{A}$, since (a) $\mathcal{B}(\mathcal{D})_{\downarrow}^{A}$ is informationally equivalent to $\mathcal{B}(\mathcal{D})^{A}$, and (b) it makes pointwise widening $\sqcap$ info-preserving. Note that $\alpha_{\cap}(X)$ is already $\preceq$-antimonotone.

Abstraction of Transition Functions. Once the abstraction $\alpha_{\cap}$ is defined, the abstraction of transition functions $\alpha_{T}$ follows from Theorem 1:

$$
\alpha_{T}\left(R^{\mathcal{C}}\right)(a) \triangleq \sqcap_{c \in \gamma_{e}(a)} \alpha_{\cap}\left(R^{\mathcal{C}}(c)\right) \quad \text { (transition abstraction) }
$$

By expanding $\alpha_{\cap}, \alpha_{T}$ can be alternatively expressed as:

$$
\begin{aligned}
& \pi_{\mathrm{t}}\left(\alpha_{T}\left(R^{\mathcal{C}}\right)(a)(b)\right)=\wedge_{c \in \gamma_{e}(a)} \operatorname{pre}\left[R^{\mathcal{C}}\right]\left(\gamma_{e}(b)\right)(c) \\
& \pi_{\mathrm{f}}\left(\alpha_{T}\left(R^{\mathcal{C}}\right)(a)(b)\right)=\wedge_{c \in \gamma_{e}(a)} \operatorname{pre} e\left[R^{\mathcal{C}}\right]\left(\neg \tilde{\gamma}_{e}(b)\right)(c)
\end{aligned}
$$

That is, if $R^{\mathcal{A}}=\alpha_{T}\left(R^{\mathcal{C}}\right)$, then a transition $R^{\mathcal{A}}(a)(b)$ between abstract states $a$ and $b$ is as true as the least degree with which all concrete states in $\gamma_{e}(a)$ have a successor in $\gamma_{e}(b)$, and as false as the least degree with which all successors of states in $\gamma_{e}(a)$ are not in $\tilde{\gamma}_{e}(b)$.

Note that so far, we have made no assumptions on the concrete transition function. However, if the concrete transition function $R^{\mathcal{C}}$ is boolean, then $R^{\mathcal{A}}=\alpha_{T}\left(R^{\mathcal{C}}\right)$ is $\mathcal{B}(\mathbf{2})$-valued and satisfies:

$$
R^{\mathcal{A}}(a)(b)=\left\langle\gamma_{e}(a) \subseteq \operatorname{pre}\left[R^{\mathcal{C}}\right]\left(\gamma_{e}(b)\right), \gamma_{e}(a) \subseteq \tilde{p r e}\left[R^{\mathcal{C}}\right]\left(\neg \tilde{\gamma}_{e}(b)\right)\right\rangle
$$

For example, let $R_{1}(x) \triangleq x+1$. A fragment of its abstraction $\alpha_{T}\left(R_{1}\right)$ is shown in Figure 2(b), where pos, neg and int are removed for clarity. For any even $x, x+$ 1 is definitely odd, but it maybe positive or negative. Thus, the transition from evn to odd is $\mathrm{d}$, and transitions to pos\&odd and to neg\&odd are $\mathrm{m}$. Note that the preimage of $\alpha_{T}\left(R_{1}\right)$ approximates the pre-image of $R_{1}$, e.g., pre $\left[\alpha_{T}\left(R_{1}\right)\right]\left(\alpha_{s}(E V E N)\right)=$ $\alpha_{s}(O D D)$.

Finally, the best abstract Kripke structure $\alpha_{\mathcal{K}}(\mathcal{C})$ of a concrete Kripke structure $\mathcal{C}=\left(C, \mathcal{D}, I^{\mathcal{C}}, R^{\mathcal{C}}\right)$ is obtained compositionally:

$$
\alpha_{\mathcal{K}}(\mathcal{C}) \triangleq\left(A, \mathcal{B}(\mathcal{D}), \alpha_{s} \circ I^{\mathcal{C}}, \alpha_{T}\left(R^{\mathcal{C}}\right)\right)
$$

(Kripke abstraction)

Thus, we were able to systematically derive rules for abstracting Kripke structures by abstract Kripke structures.

Note that the diagram in Figure 3(a) does not commute, i.e., $\alpha_{\diamond}(\operatorname{pre}[R]) \neq \operatorname{pre}\left[\alpha_{T}(R)\right]$. Thus, for a given Kripke structure, its best abstraction by an abstract $L_{\mu}$-model is more precise than its best abstraction by an abstract Kripke structure. For example, let $R_{2}$ be

$$
R_{2}(x) \triangleq \begin{cases}2 x & \text { if } x \geq 5 \wedge x \in O D D \\ -x & \text { if } 0 \leq x<5 \wedge x \in O D D \\ -2 & \text { otherwise }\end{cases}
$$


and $X \triangleq(P O S \cap E V E N) \cup(N E G \cap O D D)$. Then, $\alpha_{\diamond}\left(\operatorname{pre}\left[R_{2}\right]\right)\left(\alpha_{s}(X)\right)($ pos\&odd $)=$ $\alpha_{s}(P O S \cap O D D)($ pos\&odd $)=\mathrm{t}$, but pre $\left[\alpha_{T}\left(R_{2}\right)\right]\left(\alpha_{s}(X)\right)($ pos\&odd $)=\mathrm{m}$. This shows that transition systems are not necessarily the best abstract domain for $L_{\mu^{-}}$ preserving abstractions.

\section{Application: Abstraction of Classical Kripke Structures}

In this section, we look at boolean Kripke structures and compare our abstraction to that of Dams et al. [8], which provides an alternative way of computing the best $L_{\mu^{-}}$ preserving abstraction of Kripke structures.

We begin by addressing minor differences between the two approaches. First, the goal of [8] is to preserve satisfaction of positive $L_{\mu}$, i.e., a fragment of $L_{\mu}$ with negation restricted to atomic propositions. Second, Kripke structures are abstracted by Mixed Transition Systems (MixTSs). Essentially, a MixTS is a Kripke structure with two separate transition relations, $R^{C}$ and $R^{F}$, called constrained and free, respectively. The interpretation of $L_{\mu}$ over MixTSs is the same as its interpretation over Kripke structures, with the exception that $\diamond$ is interpreted as pre $\left[R^{C}\right]$ and $\square-$ as pre $\left[R^{F}\right]$.

Note that positive $L_{\mu}$ is as expressive as full $L_{\mu}$ : for every $L_{\mu}$ formula $\varphi$ there exists an equivalent positive formula $N N F(\varphi)$, its negation normal form. Thus, an abstraction that preserves positive $L_{\mu}$ easily extends to full $L_{\mu}$. Furthermore, the next theorem shows that MixTSs are equivalent to $\mathcal{B}(\mathbf{2})$-valued Kripke structures.

Theorem 6. Let $\mathcal{T}$ be a MixTS with statespace $A$ and transition functions $R^{C}$ and $R^{F}$, and $\mathcal{K}$ be a $\mathcal{B}(\mathbf{2})$-valued Kripke structure with the same statespace, and a transition function $R^{\mathcal{K}}$ such that $R^{\mathcal{K}}(a)(b)=\left\langle R^{C}(a)(b), \neg R^{F}(a)(b)\right\rangle$. Then, for any $L_{\mu}$ formula $\varphi,\|\varphi\|^{\mathcal{K}}=\left\langle\|N N F(\varphi)\|^{\mathcal{T}},\|N N F(\neg \varphi)\|^{\mathcal{T}}\right\rangle$.

Thus, in the case of boolean Kripke structures, the abstraction developed in this paper is equivalent to that of [8]: same structures are used as an abstract domain, and exactly the same $L_{\mu}$ formulas are preserved. However, unlike the approach taken in [8], our work systematically derives both the abstraction and the notion of abstract Kripke structures from $L_{\mu}$-preservation and the soundness relation $\rho_{s}$ between concrete and abstract sets.

It is interesting to note that although the two abstractions are equivalent w.r.t satisfaction of $L_{\mu}$, they are not identical. For completeness, Dams et al. show that the most precise MixTS abstracting a Kripke structure satisfies the following conditions:

$$
\begin{aligned}
& R^{C}(a, b) \Leftrightarrow b \in\left\{\sqcap_{y \in Y} \alpha_{e}(y) \mid Y \in \min \left\{Y^{\prime} \mid R^{\forall \exists}\left(\gamma_{e}(a), Y^{\prime}\right)\right\}\right\} \\
& R^{F}(a, b) \Leftrightarrow b \in\left\{\sqcap_{y \in Y} \alpha_{e}(y) \mid Y \in \min \left\{Y^{\prime} \mid R^{\exists}\left(\gamma_{e}(a), Y^{\prime}\right)\right\}\right\}
\end{aligned}
$$

where $R^{\forall \exists}(S, T) \triangleq \forall s \in S \cdot \exists t \in T \cdot R(s)(t)$ and $R^{\exists \exists}(S, T) \triangleq \exists s \in S \cdot \exists t \in$ $T \cdot R(s)(t)$. It is different from our abstraction $\alpha_{T}$, which, when put in this notation, is:

$$
\alpha_{T}(R)(a)(b)=\left\langle R^{\forall \exists}\left(\gamma_{e}(a), \gamma_{e}(b)\right), \neg R^{\exists \exists}\left(\gamma_{e}(a), \tilde{\gamma}_{e}(b)\right)\right\rangle
$$

We believe that our characterization is simpler; however, it remains to be seen whether it is also more useful in practice, e.g., if it leads to a smaller symbolic representation, or easier to construct compositionally, etc. We leave this topic for future work. 


\section{Related Work}

Over the years, many abstraction methods have been developed for $L_{\mu}$ model-checking $[5,8,12,17,21,22,24]$. They concentrate on a specific model - transition systems and most of them preserve soundness (satisfaction) for fragments of $L_{\mu}$ : if an abstract system is an over-approximation of the concrete one, the abstraction is sound for all universal properties. Similarly, a sound abstraction for existential properties comes from under-approximation.

The first approach for sound abstraction of full $L_{\mu}$ was proposed by Larsen and Thompsen [21]. They have shown that Modal Transition Systems (MTS) can be used to combine both over- and under-approximations. However, the goal of this work is not abstraction, and it did not consider the problem of how to abstract a Kripke structure using an MTS. The construction problem is addressed by Dams et al. [8], who independently proposed using MixTSs, a slight generalization of MTSs, as abstract models, and provided conditions for constructing an MixTS with the best precision. Although this work uses AI to describe the relationship between concrete and abstract statespaces, abstract transition systems are not derived systematically; instead, the optimal conditions are defined based on intuition, and both soundness and optimality of precision require separate proofs.

Among the attempts of using AI to systematically derive best abstractions, the work of Loiseaux et al. [22] and Schmidt [23] are the closest to ours. [22] showed how to derive a simulation-based sound abstract transition system from Galois connections within the AI framework, but their results apply only to the universal fragment of $L_{\mu}$. Motivated by the study of MixTSs, [23] showed how to capture over- and underapproximations between transition systems using AI and systematically derived Dams's most precise results. However, the starting goal of this work was formalizing the overand the under-approximations, restricting the result to the specific $L_{\mu}$ models, namely, transition systems. On the other hand, in our work we start from formalizing the notion of soundness of $L_{\mu}$ interpretations - the most general and exact goal of abstraction for $L_{\mu}$ (via the soundness relation $\rho_{i}$ in Section 4), and then systematically derive conditions which guarantee the best precision of the abstraction. Thus, our results can be applied to different $L_{\mu}$ models, where abstracting transition systems is just a special case.

Another important feature of our work is the use of bilattices. The approaches of [8, 23] develop best over- and under-approximations separately, whereas our combination of AI with bilattices provides a uniform way for abstraction of both satisfaction and refutation of $L_{\mu}$. Multi-valued logic has been previously combined with abstraction in the form of 3-valued transition systems (e.g. [16]). However, these results do not use the framework of AI, and, in particular, only deal with soundness and not the precision of the abstraction. Furthermore, 3-valued Kripke structures (unlike those based on Belnap logic) lack monotonicity [24]: a more refined abstract domain does not necessarily result in a more precise abstraction, and thus the most precise abstraction may not even exist.

\section{Conclusion}

In this paper, we have shown that abstract interpretation provides a systematic way for designing abstractions for model-checking. On one hand, our work can be seen as 
recreating the pioneering work of Dams et al. [8] in a systematic setting where each step in designing an abstraction and each loss of precision can be traced back to either the choice of an abstract domain, or the requirements on the abstract structure. On the other hand, our work also extends their results to non-traditional interpretations of $L_{\mu}$, such as its multi-valued [4] and quantitative [10] interpretations. To the best of our knowledge, this is the first abstraction technique that can be applied to these nonclassical interpretations.

In this paper, we lay the basic groundwork for designing $L_{\mu}$-preserving abstractions using the framework of AI. However, our work can be easily extended in a number of directions. We discuss a few of them below.

We have shown that requiring that an abstraction of a transition system be a transition system as well, comes with a loss of precision. Thus, it may be interesting to explore how a transition system can be abstracted directly by an abstract $L_{\mu}$ model. Such models will require new model-checking algorithms, but will provide additional precision, and possibly be easier to construct. For example, recent work on symmetry reduction [13] argues that instead of constructing a reduced abstract model, the symmetry-reduced $\diamond$ modality can be implemented directly by putting symmetry reduction inside the model-checking algorithm. We believe that our framework can be used to extend this approach to other, non-symmetry induced, abstract domains. Our work on a software model-checker YASM [18] is a first step in this direction.

In designing abstractions of Kripke structures, we have assumed that the domain and range of the transition function are abstracted by the same abstract domain. This need not be the case. By using different but related abstract domains, we obtain a generalization of "hyper-transition abstractions" [24, 11] to arbitrary abstract domains.

Although not shown explicitly in the paper, the pointwise extension of the bilattice narrowing operator $\sqcup$ to abstract structures provides a simple way to combine several, not necessarily best, abstractions. This allows us to study incremental construction of abstractions, such as the one in [1].

We believe that our framework provides an interesting starting point for exploring the connection between $\mathrm{AI}$ and model-checking, and hope to continue this line of research in the future.

\section{References}

1. T. Ball, V. Levin, and F. Xie. "Automatic Creation of Environment Models via Training". In TACAS'04, volume 2988 of $L N C S$, pages 93-107, 2004.

2. T. Ball and S. Rajamani. 'The SLAM Toolkit". In CAV'01, volume 2102 of LNCS, pages 260-264, 2001.

3. N.D. Belnap. "A Useful Four-Valued Logic". In Dunn and Epstein, editors, Modern Uses of Multiple-Valued Logic, pages 30-56. Reidel, 1977.

4. M. Chechik, B. Devereux, S. Easterbrook, and A. Gurfi nkel. 'Multi-Valued Symbolic Model-Checking". ACM TOSEM, 12(4):1-38, 2003.

5. Edmund M. Clarke, Orna Grumberg, and David E. Long. 'Model Checking and Abstraction". ACM TOPLAS, 16(5):1512-1542, 1994.

6. J. Corbett, M. Dwyer, J. Hatcliff, S. Laubach, C. Pasareanu, Robby, and H. Zheng. "Bandera: Extracting Finite-state Models from Java Source Code”. In ICSE'00, pages 439-448, 2000.

7. P. Cousot and R. Cousot. "Abstract Interpretation Frameworks". Journal of Logic and Computation, 2(4):511-547, 1992. 
8. D. Dams, R. Gerth, and O. Grumberg. "Abstract Interpretation of Reactive Systems". ACM TOPLAS, 2(19):253-291, 1997.

9. B.A. Davey and H.A. Priestley. Introduction to Lattices and Order. Cambridge University Press, 1990.

10. L. de Alfaro, M. Faella, T. A. Henzinger, R. Majumdar, and M. Stoelinga. 'Model Checking Discounted Temporal Properties”. In TACAS'04, volume 2988 of LNCS, pages 77-92, 2004.

11. L. de Alfaro, P. Godefroid, and R. Jagadeesan. "Three-Valued Abstractions of Games: Uncertainty, but with Precision". In LICS'04, pages 170-179, 2004.

12. E. A. Emerson and A. P. Sistla. 'Symmetry and Model Checking". FMSD, 9(1-2):105-131, 1996.

13. E. A. Emerson and T. Wahl. 'Dynamic Symmetry Reduction'. In TACAS'05, volume 3440 of $L N C S$, pages 382-396, 2005.

14. M. Fitting. 'Bilattices are Nice Things". In Conference on Self-Reference, 2002.

15. M. L. Ginsberg. 'Multivalued Logics: A Uniform Approach to Reasoning in Artifi cial Intelligence". Computational Intelligence, 4(3):265-316, 1988.

16. P. Godefroid, M. Huth, and R. Jagadeesan. "Abstraction-based Model Checking using Modal Transition Systems”. In CONCUR'01, volume 2154 of LNCS, pages 426-440, 2001.

17. S. Graf and H. Saïdi. 'Construction of Abstract State Graphs with PVS'. In $C A V$ '97, volume 1254 of $L N C S$, pages 72-83, 1997.

18. A. Gurfi nkel and M. Chechik. 'Yasm: Model-Checking Software with Belnap Logic". Technical Report 533, University of Toronto, April 2005.

19. A. Gurfi nkel, O. Wei, and M. Chechik. 'Logical Abstract Interpretation". Technical Report 532, University of Toronto, September 2005.

20. D Kozen. 'Results on the Propositional $\mu$-calculus". Theoretical Computer Science, 27:334354, 1983.

21. K.G. Larsen and B. Thomsen. "A Modal Process Logic". In LICS'88, pages 203-210, 1988.

22. C. Loiseaux, S. Graf, J. Sifakis, A. Bouajjani, and S. Bensalem. 'Property Preserving Abstractions for the Verifi cation of Concurrent Systems". FMSD, 6:1-35, 1995.

23. D. A. Schmidt. "Closed and Logical Relations for Over- and Under-Approximation of Powersets". In SAS'04, volume 3148 of LNCS, pages 22-37, 2004.

24. S. Shoham and O. Grumberg. 'Monotonic Abstraction-Refi nement for CTL'. In TACAS'04, LNCS, pages 546-560, April 2004.

25. O. Wei, A. Gurfi nkel, and M. Chechik. 'Identifi cation and Counter Abstraction for Full Virtual Symmetry". In CHARME'05, volume 3725 of LNCS, 2005. 